\title{
Two-Dimensional Direction-of-Arrival Estimation for Trilinear Decomposition-Based Monostatic Cross MIMO Radar
}

\author{
Fangqing Wen and Gong Zhang \\ Nanjing University of Aeronautics and Astronautics, College of Electronics \& Engineering, Nanjing 210016, China \\ Correspondence should be addressed to Fangqing Wen; wfqzhc@163.com
}

Received 22 July 2013; Revised 5 November 2013; Accepted 5 November 2013

Academic Editor: Oleg V. Gendelman

Copyright (C) 2013 F. Wen and G. Zhang. This is an open access article distributed under the Creative Commons Attribution License, which permits unrestricted use, distribution, and reproduction in any medium, provided the original work is properly cited.

\begin{abstract}
A low complexity monostatic cross multiple-in multiple-out (MIMO) radar scheme is proposed in this paper. The minimumredundancy linear array (MRLA) is introduced in the cross radar to improve the efficiency of the array elements. The twodimensional direction-of-arrival (DOA) estimation problem links to the trilinear model, which automatically pairs the estimated two-dimensional angles, requiring neither eigenvalue decomposition of received signal covariance matrix nor spectral peak searching. The proposed scheme performs better than the uniform linear arrays (ULA) configuration under the same conditions, and the proposed algorithm has less computational complexity than that of multiple signal classification (MUSIC) algorithm. Simulation results show the effectiveness of our scheme.
\end{abstract}

\section{Introduction}

Multiple-in multiple-out (MIMO) radar is a relative new concept in radar system that received considerable attention $[1,2]$. Unlike the traditional phase-array radar, the transmit antennas of MIMO radar simultaneously transmit orthogonal waveforms and utilise multiple antennas to receive the reflected signals [3]. Angel estimation is a basic problem in MIMO radar that has been investigated in $[4,5]$. Most of the previous studies are based on the uniform arrays for the simple array geometry, such as uniform linear arrays (ULA) and uniform circular-shape and L-shape arrays. The minimum-redundancy array (MRA) is an important type of the nonuniform linear geometries, with the sensor placement designed to achieve the maximum resolution for a given number of elements by reducing the redundancy of spatial correlation lags [6]. Studies show that the MRA has better angle resolution and interference suppression than the ULA for a given number of sensors [7]. Due to the capability of spanning larger aperture, a MRA configured colocated MIMO radar is proposed for one dimensional angle estimation in [8], which has achieved desired results.

Classical angle estimation algorithms such as multiple signal classification (MUSIC) and estimation of signal parameters via rotational invariance techniques (ESPRIT) require eigenvalue decomposition (EVD) or singular value decomposition (SVD) of received signal covariance matrix. In addition, MUSIC algorithm requires spectral peak searching, which would cause computation disaster in the situation of $2 \mathrm{D}$ angle estimation. ESPRIT algorithm is sensitive to the manifold of the arrays, making it unsuitable for MRA manifolds. Another problem that how to pair the estimated parameters arises within the ESPRIT algorithm, which requires the extra computational load, and usually fails to work in lower SNR. Furthermore, the subspace based methods suppose that the number of the sources is known, which is contrary to actual applications.

Trilinear decomposition (also called PARAFAC analysis) can be thought of as a generalization of ESPRIT and joint approximate diagonalization ideas [9], which is an iterative algorithm that does not need EVD or SVD. Trilinear decomposition algorithm has been used for detection and localisation of multiple targets in a ULA based MIMO radar system [10, 11]. Simulation in [11] shows that the trilinear decomposition algorithm can achieve better performance than that of ESPRIT with bistatic MIMO radar. However, the trilinear algorithm would be lapsed with the MRA 


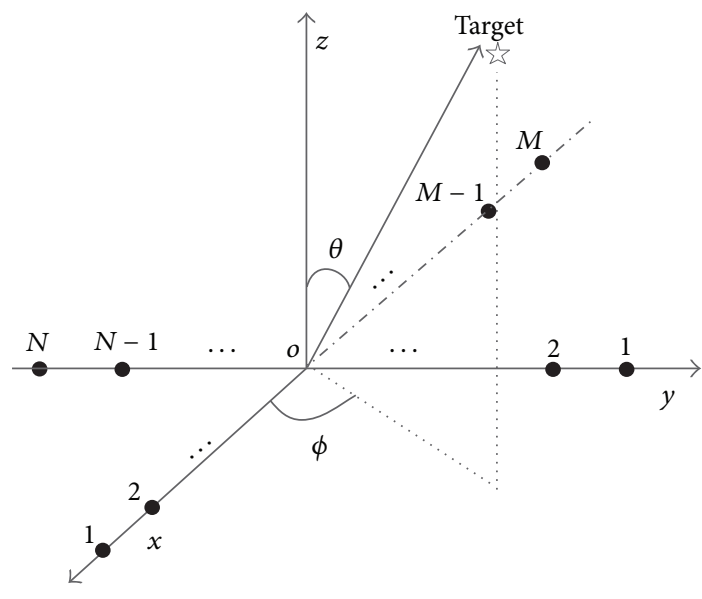

FIGURE 1: Monostatic cross MIMO radar.

configuration, as the phase ambiguity follows the received array signal, which would confuse the paired angles.

In this paper, the minimum-redundancy linear array (MRLA) configured monostatic cross MIMO radar is proposed for two-dimensional (2D) direction-of-arrival (DOA) estimation, which turned out to be effective than the ULA configuration for a given number of antennas. The improved trilinear model based blind angle estimation algorithm is developed for the proposed radar scheme. The DOA estimation problem links to the trilinear model. In order to eliminate the phase ambiguity, the estimated phase is adaptively compensated according to the array manifold. With this improvement, the proposed algorithm could be extended for arbitrarily array manifolds. We also analyse the complexity and error characteristics of the algorithm. Simulation results show the effectiveness of the proposed scheme.

The rest of the paper is organized as follows. In Section 2, we present the signal model for the monostatic cross MIMO radar. Section 3 specifies the DOA estimation method with trilinear decomposition. Simulation results are given in Section 4. Finally, we provide concluding remarks in Section 5.

Notation. Lowercase and capital letters in bold denote, respectively, vectors and matrices. The superscripts $(\bullet)^{T}$ and $(\bullet)^{H}$ represent, respectively, the transpose and Hermitian transpose of a matrix. $[\bullet, \bullet, \ldots, \bullet]$ and $[\bullet ; \bullet ; \ldots ; \bullet]$ denote arrangement of the elements in a row and in a column, respectively.

\section{Signal Model}

2.1. Monostatic Cross MIMO Radar. Consider a monostatic cross MIMO radar model, as shown in Figure 1. Without loss of generality, we suppose that $M$-element transmitter and $N$-element receiver are symmetrically located in the $x$-axis and $y$-axis. The phase center of the transmits and receivers is overlapped in the origin, while the physical location of the $m$ th transmitter and the $n$th receiver is denoted by $x_{m}$ and $y_{n}$, respectively. It is also assumed that there are $K$ noncoherent targets appearing in the far-field of the antennas with the $k$ th target at azimuth angle $\phi_{k}$ and elevation angle $\theta_{k}$. The transmit antennas transmit orthogonal waveforms with the same carrier frequency, and the transmit waveform of $m$ th antenna during $p$ th pulse is $\mathbf{s}_{m, p} \in C^{1 \times L}, p=1,2, \ldots, P$, where $P$ is the total pulse number, $L$ is the samples in a pulse period, and the reflection coefficient $\eta_{k, p}$ of the $k$ th target is assumed to be constant during the $p$ th pulse period. The received signal of the $n$th receiver $\mathbf{r}_{n, p}$ takes the form

$$
\mathbf{r}_{n, p}=\sum_{k=1}^{K} b_{n}\left(\theta_{k}, \phi_{k}\right) \eta_{k, p} \mathbf{a}^{T}\left(\theta_{k}, \phi_{k}\right) \mathbf{S}_{p}+\mathbf{e}_{n, p}
$$

where $\mathbf{a}\left(\theta_{k}, \phi_{k}\right)=\left[a_{1}\left(\theta_{k}, \phi_{k}\right), \ldots, a_{M}\left(\theta_{k}, \phi_{k}\right)\right]^{T}$ is the transmit steering vector for the $k$ th target, $\mathbf{b}\left(\theta_{k}, \phi_{k}\right)=$ $\left[b_{1}\left(\theta_{k}, \phi_{k}\right), \ldots, b_{N}\left(\theta_{k}, \phi_{k}\right)\right]^{T}$ is the corresponding receive steering vector, and $a_{m}\left(\theta_{k}, \phi_{k}\right)=e^{-j 2 \pi x_{m} \cos \phi_{k} \sin \theta_{k} / \lambda}$, $b_{n}\left(\theta_{k}, \phi_{k}\right)=e^{-j 2 \pi y_{n} \sin \phi_{k} \sin \theta_{k} / \lambda}$ with $\lambda$ denotes the wave length of the carrier. $\mathbf{S}_{p}=\left[\mathbf{s}_{1, p} ; \mathbf{s}_{2, p} ; \ldots ; \mathbf{s}_{M, p}\right]$ is the transmit wave matrix, and $\mathbf{e}_{n, p}$ is the received additive white Gaussian noise of $n$th receive antenna corresponding to the $p$ th pulse.

The received signal $\mathbf{r}_{n, p}$ is correlated with a matched filter for maximizing the signal to noise ratio (SNR) in the presence of additive stochastic noise, and the output of the matched filters corresponding to the $p$ th pulse can be expressed as

$$
\mathbf{x}_{n, p}=\sum_{l=1}^{L} \frac{\mathbf{r}_{n, p} \mathbf{S}_{p, l}^{H}}{L}
$$

where $\mathbf{S}_{p, l}$ accounts for the $l$ th column of $\mathbf{S}_{p}$.

Define $\mathbf{Q}=\left[\eta_{1,1}, \ldots, \eta_{1, P} ; \eta_{2,1} \ldots, \eta_{K, P}\right]$ as the reflection coefficients matrix, which contains the amplitude and phase information of all the $K$ targets. We suppose that the transmit waveforms fulfill

$$
\frac{1}{L} \mathbf{S}_{p} \mathbf{S}_{p}^{H}=\mathbf{I}_{M \times M} \quad(p=1, \ldots, P) .
$$

Let $\mathbf{X}_{n}=\left[\mathbf{x}_{n, 1}^{T}, \mathbf{x}_{n, 2}^{T}, \ldots, \mathbf{x}_{n, P}^{T}\right]$; then $\mathbf{X}_{n}$ can be expressed in matrix form as

$$
\mathbf{X}_{n}=\mathbf{A} D_{n}(\mathbf{B}) \mathbf{Q}+\mathbf{E}_{n} \quad(n=1, \ldots, N),
$$

where the transmit direction matrix $\mathbf{A}=\left[\mathbf{a}\left(\theta_{1}, \phi_{1}\right), \ldots\right.$, $\left.\mathbf{a}\left(\theta_{K}, \phi_{K}\right)\right] \in \mathbf{C}^{M \times K}$, the receive direction matrix $\mathbf{B}=$ $\left[\mathbf{b}\left(\theta_{1}, \phi_{1}\right), \ldots, \mathbf{b}\left(\theta_{K}, \phi_{K}\right)\right] \in \mathbf{C}^{N \times K}$, and $\mathbf{E}_{n}$ represents the interference and jamming. $D_{n}(\bullet)$ denotes the operation extracting the $n$th row of its matrix argument and constructing a diagonal matrix out of it.

2.2. Minimum-Redundancy Linear Arrays. Due to the matched filters in every receiver antenna, the transmitted waveforms can be separated from each other. The virtual elements were formed through the channel of the $m$ th transmit and the $n$th receiver, which increase the array aperture and thus improve the resolution of the MIMO radar. A uniform, linear, critically sampled array with $M=N=5$ elements is used as a reference; as depicted in Figure 2, the solid points and the hollow points represent 


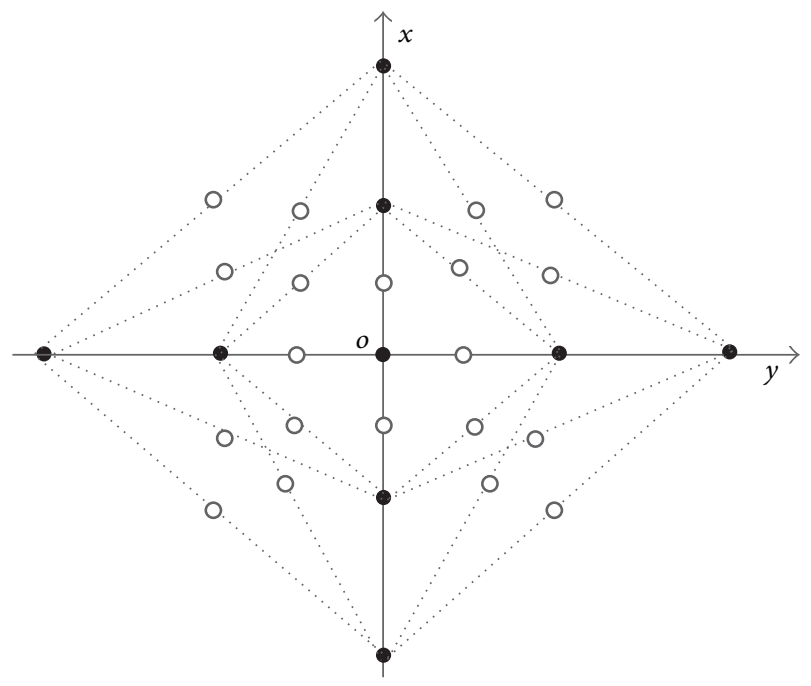

FIGURE 2: Virtual elements of cross MIMO radar.

TABLE 1: Some MRLA configurations.

\begin{tabular}{lc}
\hline Antenna number & The relative locations of antennas \\
\hline 3 & {$[-1,0,1]$} \\
4 & {$[-2,-1,1,2]$} \\
5 & {$[-3,-2,0,2,3]$} \\
6 & {$[-4,-3,-1,1,3,4]$} \\
7 & {$[-5,-4,-3,0,3,4,5]$} \\
8 & {$[-7,-6,-5,-2,1,4,5,6]$} \\
9 & {$[-8,-7,-6,-3,0,3,6,7,8]$} \\
10 & {$[-10,-9,-7,-6,-1,1,6,7,9,10]$} \\
\hline
\end{tabular}

the real elements and virtual elements, respectively. Some virtual elements overlap with real elements that are not given in the figure. Every virtual element equals a self-transmit and self-receive element, from which we can calculate that the freedom of the real aperture cross array is $M \times N$.

However, the referenced uniform linear array is redundant, which cannot utilize the elements of the array in the most efficient way. In the conventional methods, the minimum-redundancy linear array geometries together with appropriate matrix augmentation techniques have proven to provide better performances than the simple uniform linear array configuration in terms of ability to detect and resolve a greater number of sources [12]. Therefore, the transmit array and receive array of the cross MIMO radar are configured to the form of MRLA. Table 1 gives some MRLA configurations for a finite number of antennas.

2.3. Trilinear Model. The data in (4) can be rearranged as

$$
\begin{gathered}
x_{m, n, p}=\sum_{k=1}^{K} \mathbf{A}(m, k) \mathbf{B}(n, k) \mathbf{Q}(p, k)+e_{m, n, p} \\
(m=1, \ldots, M ; n=1, \ldots, N ; p=1, \ldots, P),
\end{gathered}
$$

where $e_{m, n, p}$ is the noise, $\mathbf{A}(m, k)$ stands for the $(m, k)$ th element of $\mathbf{A}$, and similarly to the others. The signal in (5) can

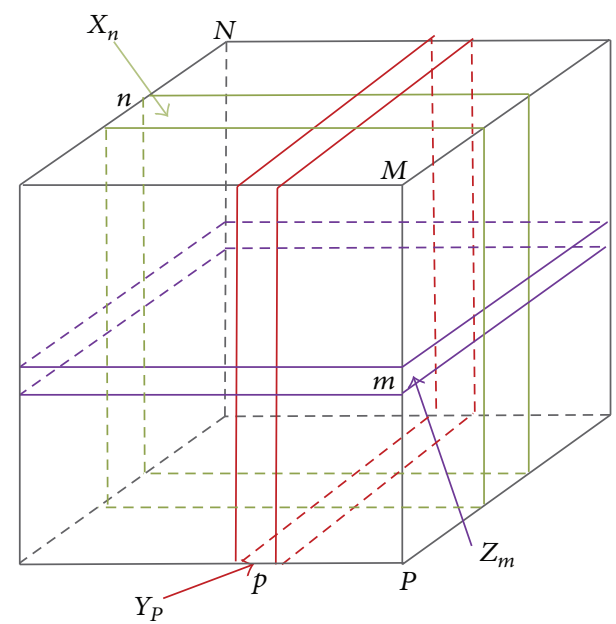

FIGURE 3: Trilinear slice model.

be denoted as the trilinear slice model, which displays the reflection for three different kinds of diversity, as shown in Figure 3. The matrix $\mathbf{X}_{n}$ can be interpreted as slicing the three dimension data in a series of slices along the spatial direction. Due to the symmetry of the trilinear model in (5), two more matrices can be interpreted as slicing the three-way data along different directions, which are denoted as

$$
\begin{array}{cc}
\mathbf{Y}_{p}=\mathbf{Q} D_{p}(\mathbf{A}) \mathbf{B}+\mathbf{E}_{p} & (p=1, \ldots, P), \\
\mathbf{Z}_{m}=\mathbf{B} D_{m}(\mathbf{Q}) \mathbf{A}+\mathbf{E}_{m} & (m=1, \ldots, M) .
\end{array}
$$

The data matrix $\mathbf{X}_{n}, \mathbf{Z}_{m}$, and $\mathbf{Y}_{p}$ are slices along the different directions when slicing the trilinear model. Rearrange the slicing data as $\mathbf{X}=\left[\mathbf{X}_{1} ; \mathbf{X}_{2} ; \ldots ; \mathbf{X}_{N}\right], \mathbf{Y}=\left[\mathbf{Y}_{1} ; \mathbf{Y}_{2} ; \ldots ; \mathbf{X}_{P}\right]$, and $\mathbf{Z}=\left[\mathbf{Z}_{1} ; \mathbf{Z}_{2} ; \ldots ; \mathbf{Z}_{M}\right]$; the problem of cross MIMO radar $2 \mathrm{D}$ angle estimation is linked to trilinear decomposition, which is also known as parallel factor analysis (PARAFAC) or canonical decomposition. It will be discussed in detail in the following section.

\section{The Trilinear Decomposition-Based 2D DOA Estimation Method}

3.1. Trilinear Alternate Least Squares. Trilinear alternating least square (TALS) algorithm is the common data detection method for trilinear model [13]. The principle of TALS can be adopted to fit low rank trilinear models. The basic idea of TALS can be described as three major steps: (a) update one of the slicing matrices $\mathbf{X}, \mathbf{Y}$, or $\mathbf{Z}$ each time using least squares (LS), which is conditioned on previously obtained estimates for the remaining matrices. (b) Proceed to update the other matrices using LS. (c) Repeat (a) and (b) until convergence of the LS cost function. TALS algorithm in our scheme is discussed as follows. 
According to (4), the LS fitting of $\mathbf{X}$ is

$$
f_{\mathbf{X}}=\min _{\mathbf{A}, \mathbf{B}, \mathbf{Q}}\left\|\left[\begin{array}{c}
\mathbf{X}_{1} \\
\mathbf{X}_{2} \\
\vdots \\
\mathbf{X}_{N}
\end{array}\right]-\left[\begin{array}{c}
\mathbf{A} D_{1}(\mathbf{B}) \\
\mathbf{A} D_{2}(\mathbf{B}) \\
\vdots \\
\mathbf{A} D_{N}(\mathbf{B})
\end{array}\right] \mathbf{Q}\right\|_{F}
$$

where $\|\bullet\|_{F}$ stands for the Frobenius norm. The LS update for $\mathbf{Q}$ is

$$
\hat{\mathbf{Q}}=\left[\begin{array}{c}
\hat{\mathbf{A}} D_{1}(\hat{\mathbf{B}}) \\
\hat{\mathbf{A}} D_{2}(\hat{\mathbf{B}}) \\
\vdots \\
\hat{\mathbf{A}} D_{N}(\hat{\mathbf{B}})
\end{array}\right]^{+}\left[\begin{array}{c}
\mathbf{X}_{1} \\
\mathbf{X}_{2} \\
\vdots \\
\mathbf{X}_{N}
\end{array}\right]
$$

where $[\bullet]^{+}$stands for the pseudoinverse and $\hat{\mathbf{A}}$ and $\hat{\mathbf{B}}$ are the matrices estimated in the last iteration.

Similarly, the LS fitting of $\mathbf{Y}$ is

$$
f_{\mathbf{Y}}=\min _{\mathbf{A}, \mathbf{B}, \mathbf{Q}}\left\|\left[\begin{array}{c}
\mathbf{Y}_{1} \\
\mathbf{Y}_{2} \\
\vdots \\
\mathbf{Y}_{P}
\end{array}\right]-\left[\begin{array}{c}
\mathbf{Q} D_{1}(\mathbf{A}) \\
\mathbf{Q} D_{2}(\mathbf{A}) \\
\vdots \\
\mathbf{Q} D_{P}(\mathbf{A})
\end{array}\right] \mathbf{B}\right\|_{F}
$$

and the LS update for $\mathbf{B}$ is

$$
\hat{\mathbf{B}}=\left[\begin{array}{c}
\hat{\mathbf{Q}} D_{1}(\hat{\mathbf{A}}) \\
\hat{\mathbf{Q}} D_{2}(\hat{\mathbf{A}}) \\
\vdots \\
\hat{\mathbf{Q}} D_{P}(\hat{\mathbf{A}})
\end{array}\right]^{+}\left[\begin{array}{c}
\mathbf{Y}_{1} \\
\mathbf{Y}_{2} \\
\vdots \\
\mathbf{Y}_{P}
\end{array}\right],
$$

where $\hat{\mathbf{Q}}$ and $\hat{\mathbf{A}}$ stand for the previously obtained estimation of $\mathbf{Q}$ and $\mathbf{A}$.

Finally, the LS fitting of $\mathbf{Z}$ is

$$
f_{\mathbf{Z}}=\min _{\mathbf{A}, \mathbf{B}, \mathbf{Q}}\left\|\left[\begin{array}{c}
\mathbf{Z}_{1} \\
\mathbf{Z}_{2} \\
\vdots \\
\mathbf{Z}_{M}
\end{array}\right]-\left[\begin{array}{c}
\mathbf{B} D_{1}(\mathbf{Q}) \\
\mathbf{B} D_{2}(\mathbf{Q}) \\
\vdots \\
\mathbf{B} D_{M}(\mathbf{Q})
\end{array}\right] \mathbf{A}\right\|_{F}
$$

and the LS update for $\mathbf{A}$ is

$$
\hat{\mathbf{A}}=\left[\begin{array}{c}
\hat{\mathbf{B}} D_{1}(\hat{\mathbf{Q}}) \\
\hat{\mathbf{B}} D_{2}(\hat{\mathbf{Q}}) \\
\vdots \\
\hat{\mathbf{B}} D_{M}(\hat{\mathbf{Q}})
\end{array}\right]^{+}\left[\begin{array}{c}
\mathbf{Z}_{1} \\
\mathbf{Z}_{2} \\
\vdots \\
\mathbf{Z}_{M}
\end{array}\right]
$$

where $\hat{\mathbf{B}}$ and $\hat{\mathbf{Q}}$ stand for the previously obtained estimates of $\mathbf{B}$ and $\mathbf{Q}$.
Although TALS algorithm is easy to implement and guarantee to converge, it also suffers from slow convergence. In [14] the COMFAC algorithm is proposed to speed up the LS fitting of TALS. COMFAC compresses the high dimensional three-way array into the subspaces defined by the three bases of $\mathbf{A}, \mathbf{B}$, and $\mathbf{Q}$. After fitting the PARAFAC model in the compressed space, the solution is recovered to the original space, which is followed by a few TALS steps in the uncompressed space. For noise-free data with no model error an exact solution can be calculated directly by posing the fitting problem as a generalized eigenvalue problem. With the presence of the noise, the COMFAC algorithm gave the LS estimation of $\mathbf{A}, \mathbf{B}$, and $\mathbf{Q}$. In this paper, the COMFAC algorithm is selected for trilinear decomposition.

3.2. Uniqueness and Identifiability Analysis. Just as singular value decomposition (SVD) is a decomposition of twoway arrays into rank one two-way components, trilinear decomposition is a decomposition of three-way arrays into rank one three-way components. The fundamental difference is that the trilinear decomposition is unique under certain rank conditions, whereas bilinear decompositions into rank one components are not unique without imposing additional constraints. According to [15], the trilinear decomposition of the noiseless three-way array $\mathbf{X}_{n},(n=1, \ldots, N)$ is unique if

$$
k_{\mathrm{A}}+k_{\mathrm{B}}+k_{\mathbf{Q}} \geq 2 K+2,
$$

where $k_{\mathrm{A}}, k_{\mathrm{B}}$, and $k_{\mathrm{Q}}$ are $k$-rank of the corresponding matrices and $K$ is the rank of $\mathbf{X}_{n}$. A matrix has $k$-rank equal to $k$ if any subset of $k$ columns are linearly independent and there exists a subset of $k+1$ linearly dependent columns. When the uniqueness condition (13) is satisfied, trilinear decomposition of the received three-way array $\mathbf{X}_{n}$ can be used to uniquely recover matrices $\mathbf{A}, \mathbf{B}$, and $\mathbf{Q}$ up to permutation and scaling of their columns. With the noisy received signal, any other triple $\overline{\mathbf{A}}, \overline{\mathbf{B}}$, and $\overline{\mathbf{Q}}$ that construct $\mathbf{X}_{n},(n=1, \ldots, N)$ is related to $\mathbf{A}, \mathbf{B}$, and $\mathbf{Q}$ via

$$
\begin{gathered}
\overline{\mathbf{A}}=\mathrm{A} \Pi \Delta_{1}+\mathbf{N}_{1}, \quad \overline{\mathbf{B}}=\mathbf{B} \Pi \Delta_{2}+\mathbf{N}_{2}, \\
\overline{\mathbf{Q}}=\mathbf{Q} \Pi \Delta_{3}+\mathbf{N}_{3},
\end{gathered}
$$

where $\boldsymbol{\Pi}$ is a permutation matrix, $\mathbf{N}_{1}, \mathbf{N}_{2}$, and $\mathbf{N}_{3}$ are the corresponding estimation errors, which is closely related to the SNR. $\Delta_{1}, \Delta_{2}$, and $\Delta_{3}$ are diagonal scaling matrix with $\Delta_{1} \Delta_{2} \Delta_{3}=$ I.

When the matrix $k_{\mathrm{A}}$ and $k_{\mathrm{B}}$ are Vandermonde matrices with distinct nonzero generators, and $\mathbf{Q} \in \mathbf{C}^{K \times P}$ is full $k$-rank, (13) becomes

$$
M+N+\min \{K, P\} \geq 2 K+2,
$$

and in general we have $P \geq K$; the maximal number of targets that the proposed algorithm can detect is $M+N-2$.

3.3. 2D DOA Estimation. Once trilinear decomposition is accomplished, the estimated direction matrices $\widehat{\mathbf{A}}$ and $\widehat{\mathbf{B}}$ are obtained, and then the 2D DOA can be estimated with LS 
method. The estimated transmit vector $\mathbf{a}\left(\theta_{k}, \phi_{k}\right)$ was firstly normalized to the reference phase center. Define

$$
\mathbf{h}_{1}=-\operatorname{angle}\left[\hat{\mathbf{a}}\left(\theta_{k}, \phi_{k}\right)\right]+\pi \mathbf{w}_{1},
$$

where $\mathbf{w}_{\mathbf{1}}=\left[w_{1,1}, w_{1,2}, \ldots, w_{1, M}\right]$ with $w_{1, m} \in N(m=$ $1,2, \ldots, M)$. As the phase ambiguity exits the MRLA configured receive signal, the added $w_{1, m} \pi$ makes sure that $\mathbf{h}_{1}$ is an ascending sequence. Let

$$
\mathbf{P}_{1}=\left[\begin{array}{cc}
1 & x_{1} \\
1 & x_{2} \\
\vdots & \vdots \\
1 & x_{M}
\end{array}\right], \quad \mathbf{u}_{1}=\left[\begin{array}{l}
u_{1} \\
u_{2}
\end{array}\right]
$$

and define $\xi_{k}=2 \pi \cos \phi_{k} \sin \theta_{k} / \lambda$; it is obvious that $u_{2}$ is the LS estimation of $\xi_{k}$. The LS solution for $\mathbf{u}$ is

$$
\mathbf{u}=\left(\mathbf{P}_{1}^{T} \mathbf{P}_{1}\right)^{-1} \mathbf{P}_{1}^{T} \mathbf{h}_{1}
$$

Similarity, define

$$
\begin{gathered}
\mathbf{h}_{2}=-\operatorname{angle}\left[\hat{\mathbf{b}}\left(\theta_{k}, \phi_{k}\right)\right]+\pi \mathbf{w}_{2}, \\
\mathbf{P}_{2}=\left[\begin{array}{cc}
1 & y_{1} \\
1 & y_{2} \\
\vdots & \vdots \\
1 & y_{N}
\end{array}\right]\left[\begin{array}{cc}
1 & y_{1} \\
1 & y_{2} \\
\vdots & \vdots \\
1 & y_{N}
\end{array}\right], \quad \mathbf{v}=\left[\begin{array}{l}
v_{1} \\
v_{2}
\end{array}\right] .
\end{gathered}
$$

where $v_{2}$ is the LS estimation of $\zeta_{k}, \zeta_{k}=2 \pi \sin \phi_{k} \sin \theta_{k} / \lambda$. The LS solution for $\mathbf{v}$ is

$$
\mathbf{v}=\left(\mathbf{P}_{2}^{T} \mathbf{P}_{2}\right)^{-1} \mathbf{P}_{2}^{T} \mathbf{h}_{2}
$$

The azimuth angle and elevation angle can be paired automatically through the following formula:

$$
\begin{aligned}
& \boldsymbol{\theta}_{k}=\arcsin \left(\frac{\lambda}{2 \pi} \sqrt{u_{2}^{2}+v_{2}^{2}}\right), \\
& \phi_{k}=\arctan \left(\frac{v_{2}}{u_{2}}\right) .
\end{aligned}
$$

3.4. Scheme Analysis. Traditional Capon and MUSIC algorithm requires $2 \mathrm{D}$ peak searching, which brings very heavy complexity. Others are effective only in condition that transmit and receive are uniform arrays. However, the proposed trilinear decomposition-based algorithm is effective for manifold of the ULA and MRLA. Compared with ESPRIT, which process the complexity of $O\left(M^{2} N^{2} P+M^{3} N^{3}+K^{3}\right)$ [11], the complexity of each TALS iteration is $O\left(K^{3}+M N P K\right)$; generally $K$ is relatively small compared to $M, N$, and $P$, and only a few iterations are required to achieve convergence.

According to [16], the Cramér-Rao Bound (CRB) of the estimated error can be derived as

$$
\mathrm{CRB}=\frac{\sigma^{2}}{2}\left\{\operatorname{Re}\left[\left(\mathbf{D}^{H} \boldsymbol{\Pi}_{\mathbf{F}}^{\perp} \mathbf{D}\right) \odot \mathbf{W}^{T}\right]\right\}^{-1},
$$

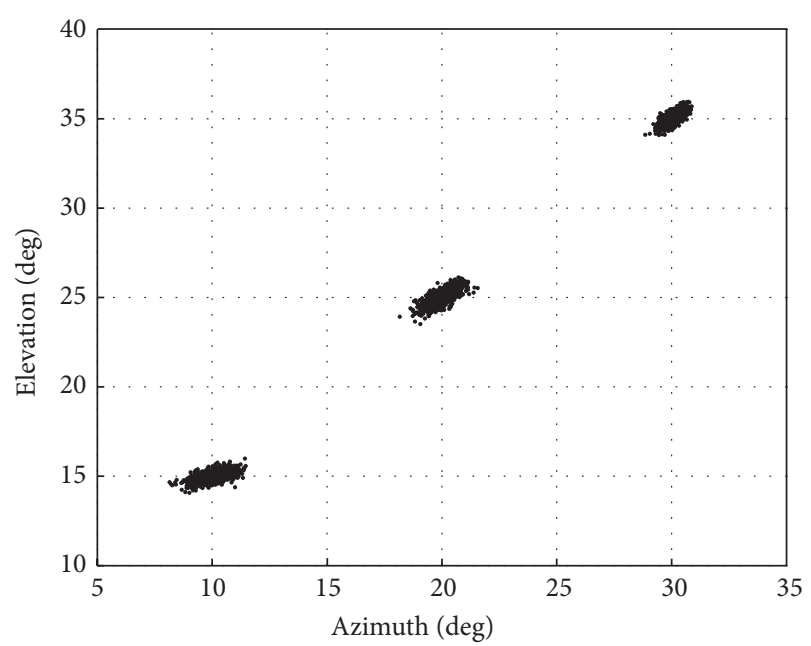

Figure 4: Angle estimation results with $\mathrm{SNR}=-5 \mathrm{~dB}$.

where $\mathbf{F}$ is defined as $\mathbf{F}=\mathbf{A} \otimes \mathbf{B}=\left[\mathbf{f}_{1}, \ldots, \mathbf{f}_{K}\right]$ and $\odot$ and $\otimes$ represent Hadamard product and Kronecker product, respectively. $\sigma$ is the noise level after the matched filtering. $\mathbf{D}=\left[\partial \mathbf{f}_{1} / \theta_{1}, \ldots, \partial \mathbf{f}_{K} / \theta_{K}, \partial \mathbf{f}_{1} / \phi_{1}, \ldots, \partial \mathbf{f}_{K} / \phi_{K}\right], \Pi_{\mathbf{F}}^{\perp}=\mathbf{I}_{M \times N}-$ $\mathbf{F}\left(\mathbf{F}^{H} \mathbf{F}\right)^{-1} \mathbf{F}^{H}$, and $\mathbf{W}=\left[\mathbf{P}_{w}^{T}, \mathbf{P}_{w}^{T}\right]^{T}$ with $\mathbf{P}_{w}=\left[\mathbf{P}_{s}, \mathbf{P}_{s}\right]$ and $\mathbf{P}_{s}=(1 / P) \mathbf{Q} \mathbf{Q}^{H}$.

\section{Simulation Results}

To assess the angle estimation performance of the proposed algorithm, 1000 Monte Carlo simulations are presented. The root mean squared error (RMSE) of 2D-DOD is defined as follows:

$$
\mathrm{RMSE}=\frac{1}{K} \sum_{k=1}^{K} \sqrt{\sum_{i=1}^{1000}\left(\hat{\theta}_{i, k}-\theta_{k}\right)^{2}+\left(\hat{\phi}_{i, k}-\phi_{k}\right)^{2}}
$$

where $\hat{\theta}_{i, k}$ and $\hat{\phi}_{i, k}$ denote the estimation of azimuth and elevation angle of the $k$ th target in the $i$ th Monte Carlo trial. Note that $M, N, P$, and $K$ are the number of transmit antennas, the receive antennas, the pulse, and targets, respectively. The reflection coefficients of the targets are assumed to fulfill the classic Swerling Case 2. The SNR is defined before the matched filtering. We assume that there are three uncorrelated targets located at angles $\left(\theta_{1}, \phi_{1}\right)=\left(10^{\circ}, 15^{\circ}\right)$, $\left(\theta_{2}, \phi_{2}\right)=\left(20^{\circ}, 25^{\circ}\right)$, and $\left(\theta_{3}, \phi_{3}\right)=\left(30^{\circ}, 35^{\circ}\right)$, respectively. $M=N=5$ and the manifold of the transmit and receive antennas are referenced in Table 1 . The samples during a pulse are designated to $L=128$.

Figures 4 and 5 present the estimation results of the proposed scheme with $\mathrm{SNR}=-5 \mathrm{~dB}$ and $5 \mathrm{~dB}$ separately and with $P=256$ considered in the simulation. It is observed that the proposed scheme is able to estimate azimuth angle and elevation angle for the radar system.

We compare the proposed algorithm and 2D MUSIC algorithm with $P=256$, and the searching intervals of 2D MUSIC algorithm are all $0.1^{\circ}$. Figure 6 presents the RMSE of the estimation results. We find that the RMSE 


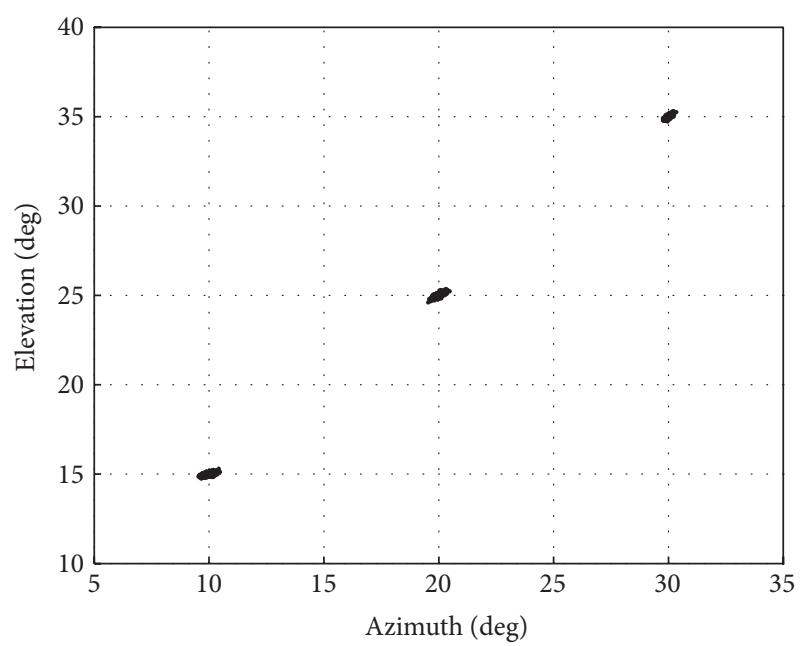

FIgUre 5: Angle estimation results with $\mathrm{SNR}=5 \mathrm{~dB}$.

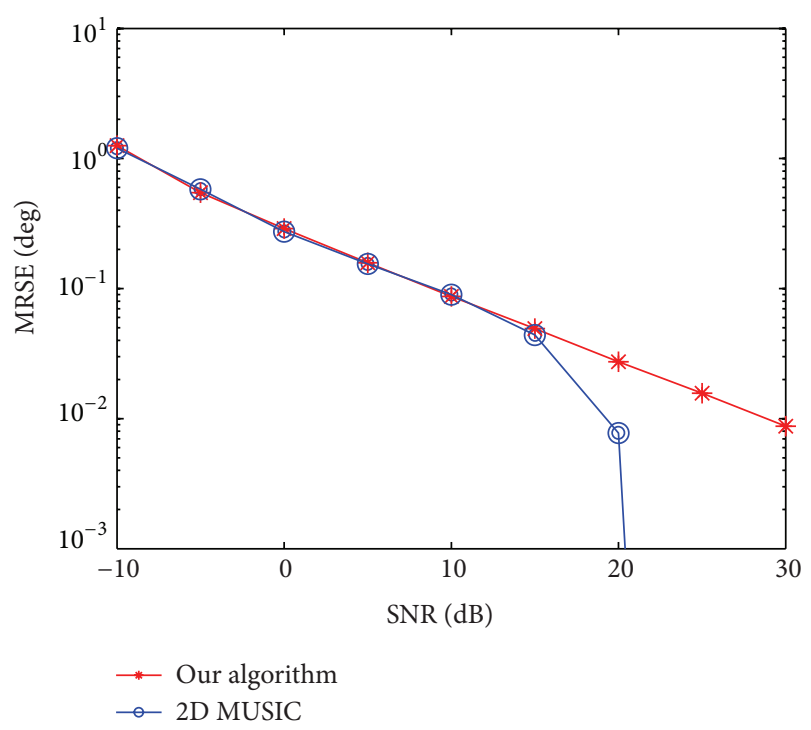

Figure 6: RMSE comparison with 2D MUSIC.

TABLE 2: Average programme time comparison.

\begin{tabular}{lcccc}
\hline Algorithm SNR & $-5 \mathrm{~dB}$ & $0 \mathrm{~dB}$ & $5 \mathrm{~dB}$ & $10 \mathrm{~dB}$ \\
\hline 2D MUSIC & $6.476 \mathrm{~s}$ & $6.479 \mathrm{~s}$ & $6.529 \mathrm{~s}$ & $6.482 \mathrm{~s}$ \\
Proposed algorithm & $0.236 \mathrm{~s}$ & $0.228 \mathrm{~s}$ & $0.227 \mathrm{~s}$ & $0.226 \mathrm{~s}$ \\
\hline Algorithm SNR & $15 \mathrm{~dB}$ & $20 \mathrm{~dB}$ & $25 \mathrm{~dB}$ & $30 \mathrm{~dB}$ \\
\hline 2D MUSIC & $6.498 \mathrm{~s}$ & $6.559 \mathrm{~s}$ & $6.578 \mathrm{~s}$ & $6.508 \mathrm{~s}$ \\
Proposed algorithm & $0.224 \mathrm{~s}$ & $0.224 \mathrm{~s}$ & $0.226 \mathrm{~s}$ & $0.224 \mathrm{~s}$ \\
\hline
\end{tabular}

performance of the proposed algorithm is very close to that of the 2D MUSIC algorithm (due to the limited searching grid, the performance of MUSIC algorithm seems better than the proposed algorithm with higher SNR). Table 2 shows the average time the algorithms run. It is obvious that the
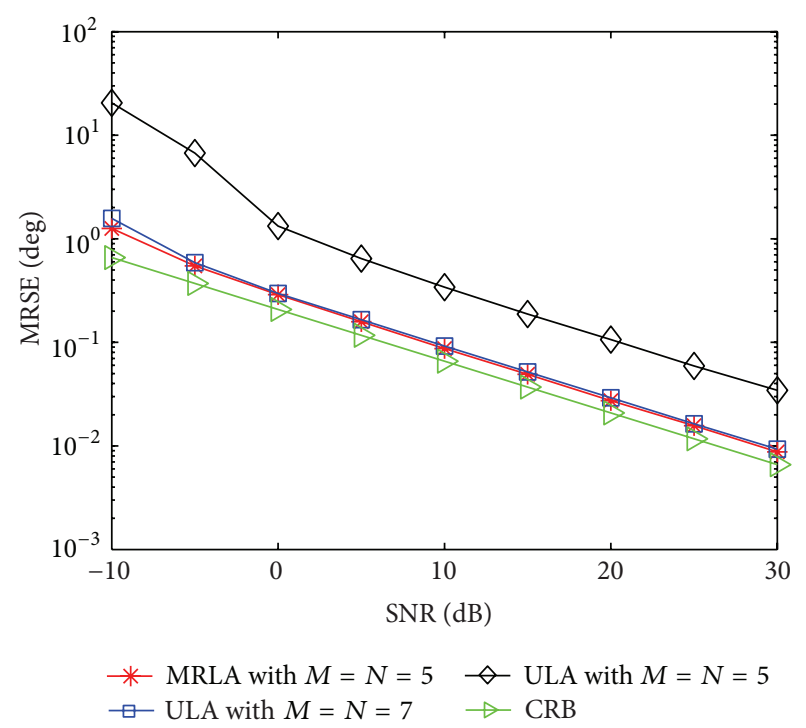

FIGURE 7: RMSE comparison with ULA.

proposed algorithm processes a higher operating efficiency. The PARAFAC algorithm is iterative and good starting values are beneficial in keeping computation time low [14], but it is hard for the algorithm to achieve a perfect initial value with lower SNR. The simulation results also prove that the low SNR would affect the convergence speed of TALS.

Figure 7 depicts the angle estimation performance comparison of the proposed MRLA based cross MIMO radar and the ULA configuration with $M=N=5$ and $M=N=7$, respectively. Simulation results agree with what we have expected, and the performance of MRLA configuration is very close to that of the equivalent ULA configuration.

The performance of the proposed scheme under different pulse number $P$ is investigated in the simulation, as shown in Figure 8. It indicates that angle estimation performance of this algorithm is improved with snapshot number $P$ increasing, but after the snapshot reaches a level, this improvement slows down. This phenomenon can be interpreted from (22). That means that the estimated error is affected by the noise variance $\sigma^{2}$ and more snapshots mean more accurate estimation of the noise. The estimation error would tend to stable $(P \geq 256)$ with sufficient snapshot number.

We also compared the RMSE performance of the cross radar with MRLA and ULA configuration, as shown in Figure 9 and $M=N$ is considered in the simulation. It is indicated that the angle estimation performance of our algorithm is gradually improved with the increasing number of antennas. Multiple antennas improve estimation performance because of diversity gain. Compared with the ULA configuration with the same number of antenna, the MRLA manifold brings much more virtual elements and thus achieves much better estimation performance. 


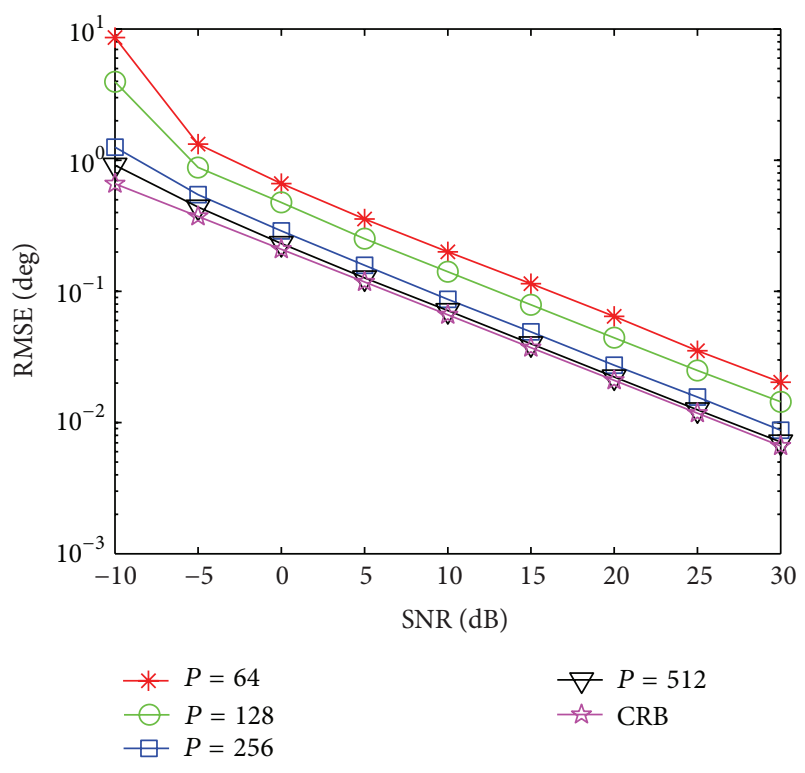

FIGURE 8: RMSE comparison with different $P$.

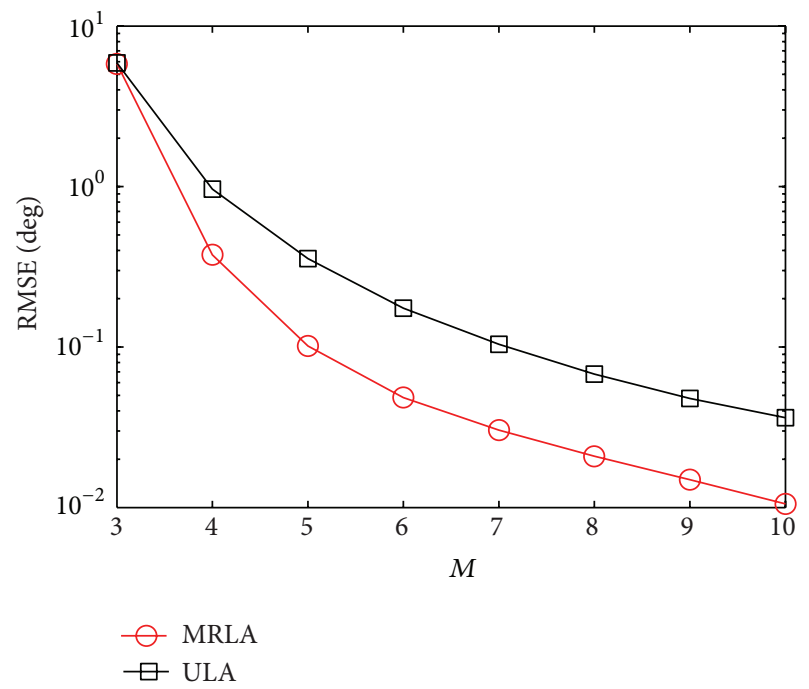

FIGURE 9: RMSE comparison with different $M, N$.

\section{Conclusion}

In this paper, we proposed the MRLA scheme for monostatic cross MIMO radar, and the trilinear decomposition algorithm is developed for two-dimensional angle estimation. Without eigenvalue decomposition of received signal covariance matrix and peak searching, the proposed algorithm can achieve automatic pairing two-dimensional angles and works well. Furthermore, our scheme has better performance than the ULA based scheme and has a very close performance to 2D MUSIC algorithm, which has heavier computational complexity than the proposed algorithm. Simulation results proved that the proposed scheme would achieve super performance in radar parameter estimation.

\section{Conflict of Interests}

The authors declare that there is no conflict of interests regarding the publication of this paper.

\section{Acknowledgments}

This work was supported by the Chinese National Natural Science Foundation under Contract nos. 61071163, 61071164, 61271327, and 61201367 and partly funded by the Priority Academic Program Development of Jiangsu Higher Education Institutions (PADA).

\section{References}

[1] L. Xu, J. Li, and P. Stoica, "Radar imaging via adaptive mimo techniques," in Proceedings of the 14th European Signal Processing Conference (EUSIPCO '06), EURASIP, Florence, Italy, September 2006.

[2] J. Li, P. Stoica, L. Xu, and W. Roberts, "On parameter identifiability of MIMO radar," IEEE Signal Processing Letters, vol. 14, no. 12, pp. 968-971, 2007.

[3] E. Fishler, A. Haimovich, R. Blum, D. Chizhik, L. Cimini, and R. Valenzuela, "MIMO radar: an idea whose time has come," in Proceedings of the IEEE Radar Conference, pp. 71-78, IEEE, April 2004.

[4] M. L. Bencheikh, Y. Wang, and H. He, "Polynomial root finding technique for joint DOA DOD estimation in bistatic MIMO radar," Signal Processing, vol. 90, no. 9, pp. 2723-2730, 2010.

[5] Y. Cheng, R. Yu, H. Gu, and W. Su, "Multi-svd based subspace estimation to improve angle estimation accuracy in bistatic mimo radar," Signal Processing, 2013.

[6] H. Duan, B. P. Ng, C. M. S. See, and J. Fang, "Broadband interference suppression performance of minimum redundancy arrays," IEEE Transactions on Signal Processing, vol. 56, no. 6, pp. 2406-2416, 2008.

[7] H. Duan, B. P. Ng, C. M. S. See, and J. Fang, "Spatial resolutions of the broadband nonredundant and minimum redundancy arrays," IEEE Signal Processing Letters, vol. 14, no. 11, pp. 852855, 2007.

[8] Z. Peng, D. Ben, G. Zhang, and H. Gu, "Non-uniform linear array configuration for MIMO radar," Transactions of Nanjing University of Aeronautics and Astronautics, vol. 28, no. 2, pp. 152-156, 2011.

[9] L. De Lathauwer, "A link between the canonical decomposition in multilinear algebra and simultaneous matrix diagonalization," SIAM Journal on Matrix Analysis and Applications, vol. 28, no. 3, pp. 642-666, 2006.

[10] D. Nion and N. D. Sidiropoulos, "A Parafac-based technique for detection and localization of multiple targets in a MIMO radar system," in Proceedings of the IEEE International Conference on Acoustics, Speech, and Signal Processing (ICASSP '09), pp. 20772080, IEEE, April 2009.

[11] X. Zhang, Z. Xu, L. Xu, and D. Xu, “Trilinear decompositionbased transmit angle and receive angle estimation for multipleinput multiple-output radar," IET Radar, Sonar and Navigation, vol. 5, no. 6, pp. 626-631, 2011.

[12] G. Gelli and L. Izzo, "Minimum-redundancy linear arrays for cyclostationarity-based source location," IEEE Transactions on Signal Processing, vol. 45, no. 10, pp. 2605-2608, 1997. 
[13] N. D. Sidiropoulos, G. B. Giannakis, and R. Bro, "Blind parafac receivers for DS-CDMA systems," IEEE Transactions on Signal Processing, vol. 48, no. 3, pp. 810-823, 2000.

[14] R. Bro, N. D. Sidiropoulos, and G. B. Giannakis, "A fast least squares algorithm for separating trilinear mixtures," in Workshop on Independent Component Analysis for Blind Signal Separation, pp. 289-294, 1999.

[15] N. D. Sidiropoulos and X. Liu, "Identifiability results for blind beamforming in incoherent multipath with small delay spread," IEEE Transactions on Signal Processing, vol. 49, no. 1, pp. 228236, 2001.

[16] P. Stoica and A. Nehorai, "Performance study of conditional and unconditional direction-of-arrival estimation," IEEE Transactions on Acoustics, Speech, and Signal Processing, vol. 38, no. 10, pp. 1783-1795, 1990. 


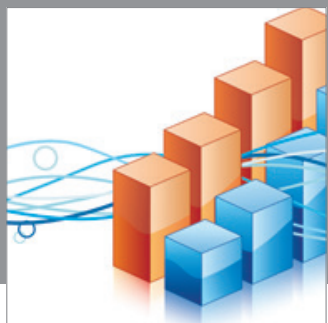

Advances in

Operations Research

mansans

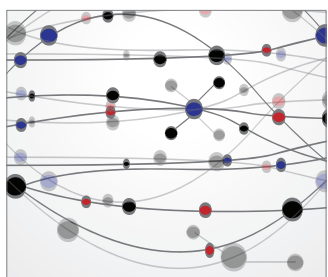

The Scientific World Journal
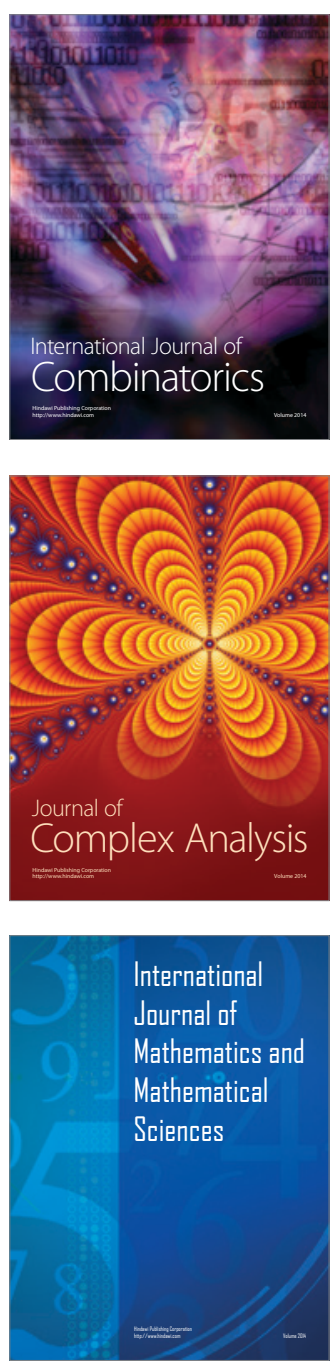
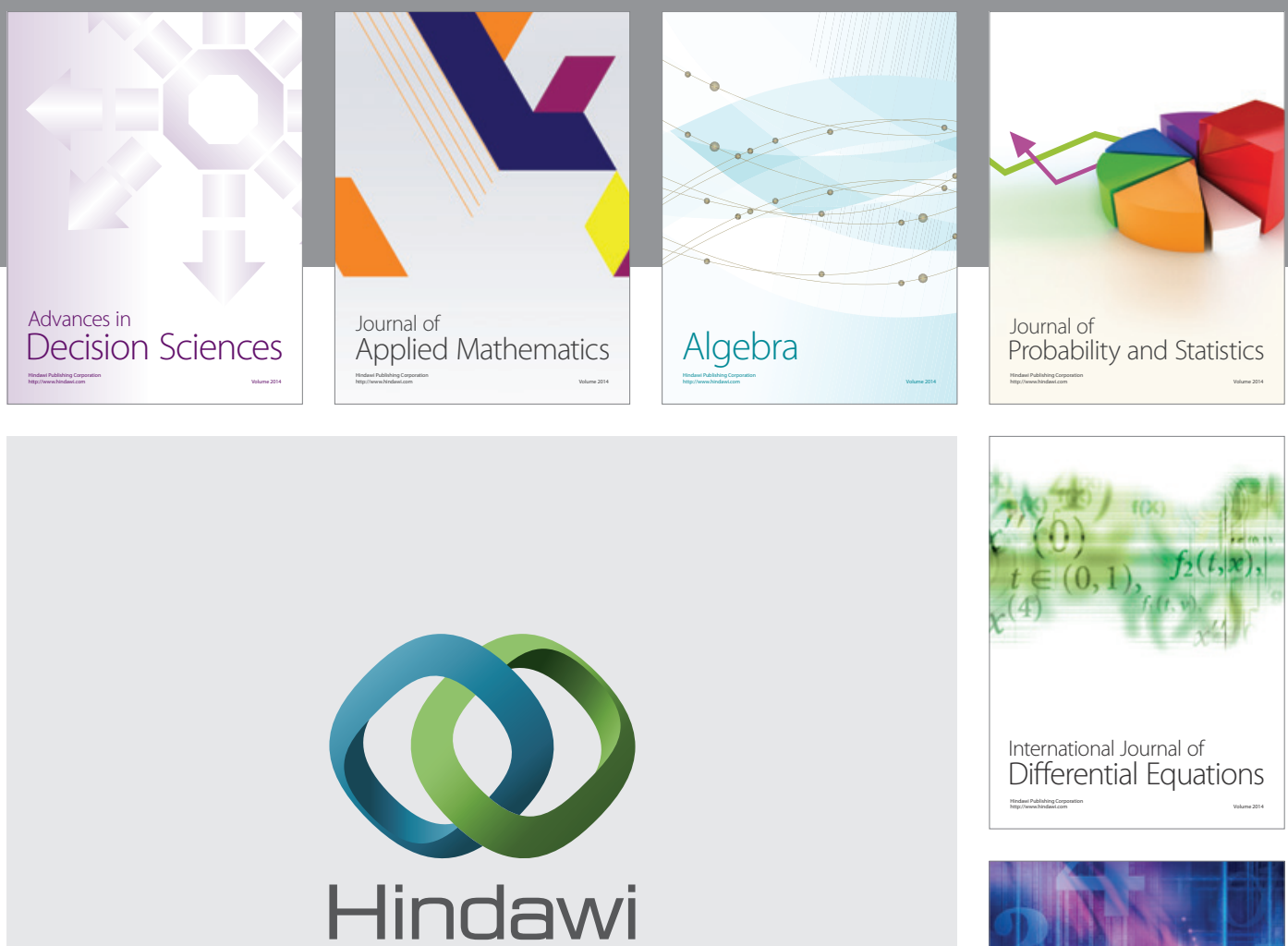

Submit your manuscripts at http://www.hindawi.com
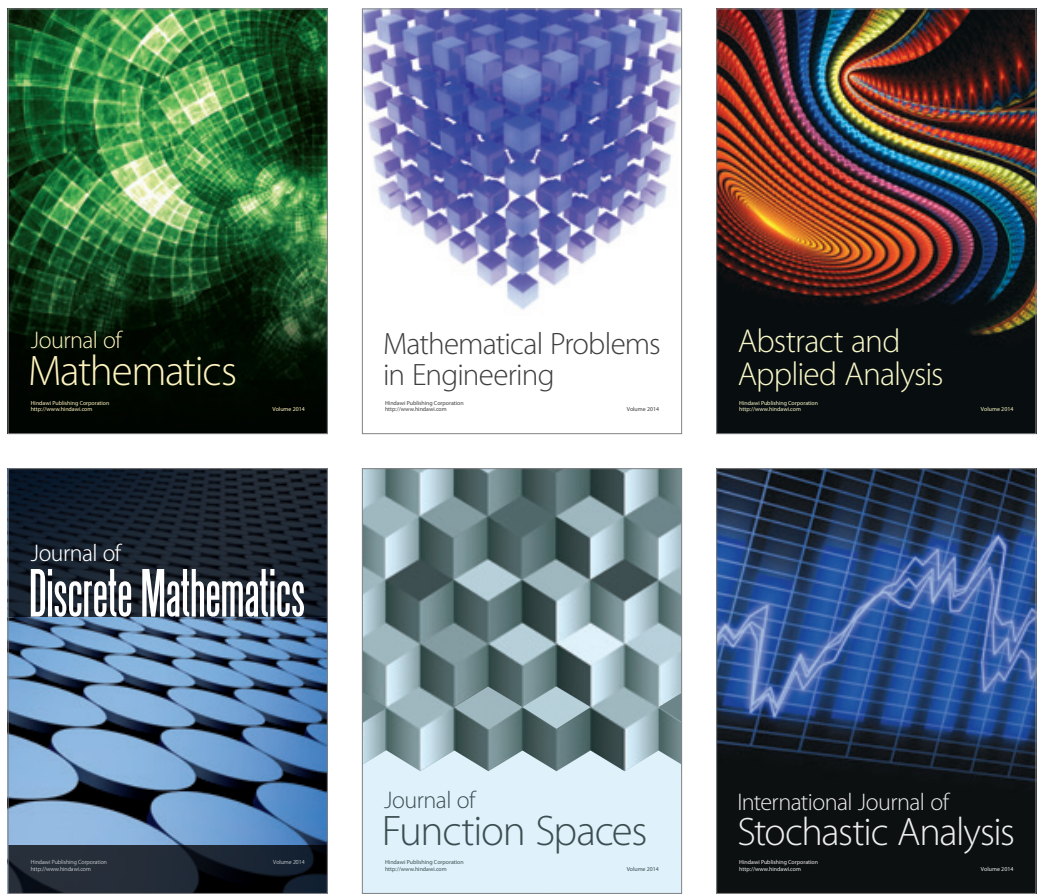

Journal of

Function Spaces

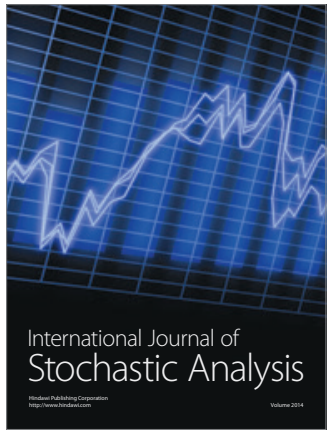

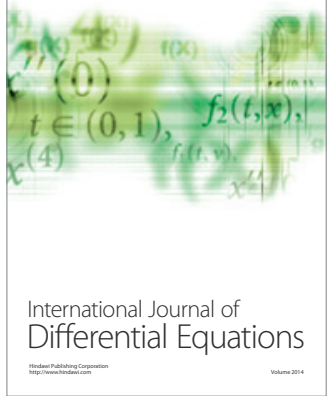
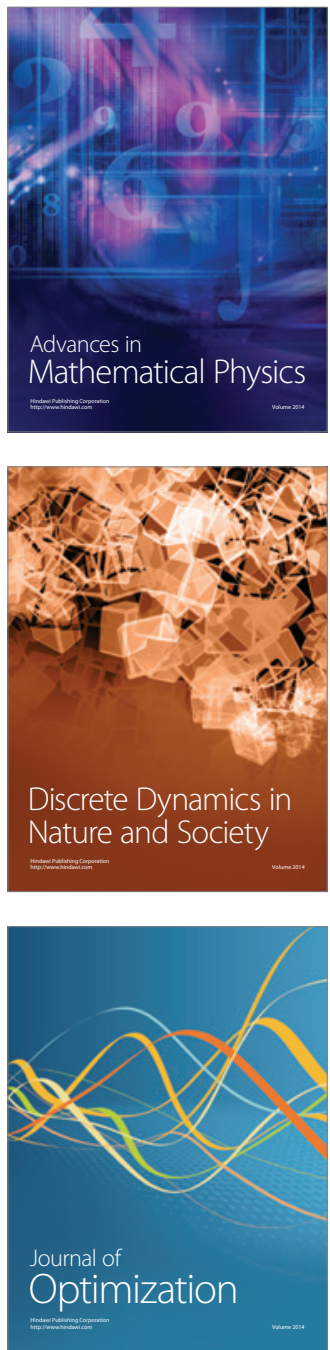